\section{PWE-011 DIAGNOSING ABDOMINAL TUBERCULOSIS IN THE ACUTE ABDOMEN}

${ }^{1} \mathrm{KA}$ Burke*, ${ }^{2} \mathrm{~K}$ Thiruppathy, ${ }^{3} \mathrm{~A}$ Patel, ${ }^{4} \mathrm{~A}$ Jayaratnam, ${ }^{2} \mathrm{~S}$ Snooks. ${ }^{1}$ Barts and the London School of Medicine and Dentistry, London; ${ }^{2}$ Department of General Surgery, King Geroge Hospital, Essex, UK; ${ }^{3}$ University of Southampton, Southampton; ${ }^{4}$ King George Hospital, Essex, UK

\subsection{6/gutjpl-2014-307263.271}

Introduction Despite recent improvements in medical treatments, the incidence of abdominal tuberculosis (ATB) in the United Kingdom has increased over the past two decades. This case series examined the difficulties encountered in correctly diagnosing this infection.

Methods A retrospective study was undertaken, reviewing the records of 36 patients diagnosed with ATB from 2000 to 2012 at a district general hospital in outer East London.

Results The commonest presenting feature was abdominal pain in $67(\%)$ of patients, and the most common sites of infection were the iliocaecal junction and peritoneum, seen in 36.1 (\%) and $33.3(\%)$ respectively. Six patients were initially investigated for Crohn's disease and one for ileitis. The highest disease prevalence was seen in patients born in India and Pakistan, which was $27.8(\%)$ and $19.4(\%)$ of patients respectively.

Colonoscopy was performed in nine patients, and three of these reported normal findings. The other six reported visible non-specific inflammatory changes. Three patients had abdominal x-rays reported and one patient had an abdominal ultrasound, all of which were normal. An abdominal computerised topography (CT) scan was performed in 26 patients and a chest CT was undertaken in 19 patients. Varying degrees of inflammatory changes were seen in all of the patients who had CT scans. Microbiological culture was positive for mycobacterium tuberculosis or acid-fast bacilli in $71(\%)$ of patients. Conclusion Abdominal tuberculosis can be very difficult to diagnose as symptoms are non-specific and can mimic other types of granulomatous inflammatory bowel diseases. Radiology appears largely unhelpful due to the non-specificity of any positive imaging findings, and there is a lack of diagnostic procedural and microbiological tests with high specificity and sensitivity. In view of the increasing incidence of tuberculosis in the United Kingdom, there should be a high index of suspicion for ATB in individuals from high-incidence countries who present with nonspecific abdominal symptoms.

\section{REFERENCES}

Uzunkoy A, Harma M, Harma M. Diagnosis of abdominal tuberculosis: Experience from 11 cases and review of the literature. World Journal of Gastroenterology 2004:10(24):3547-3549

Rai S, Thomas W M Diagnosis of abdominal tuberculosis: the importance of laparoscopy. Journal of the Royal Society of Medicine 2003;96:586-588

National Institute for Health and Clinical Excellence Tuberculosis. Clinical diagnosis and management of tuberculosis, and measures for its prevention and control. NICE clinical guideline 2011;117

Disclosure of Interest None Declared.

\section{PWE-012 AUDIT OF NHS TAYSIDE COLONOSCOPY SURVEILLANCE PROGRAMME}

${ }^{1} \mathrm{~K}$ Elliott ${ }^{*},{ }^{2} \mathrm{~L}$ Gray, ${ }^{3} \mathrm{M}$ Groome, ${ }^{2} \mathrm{~J}$ Rodger, ${ }^{3} \mathrm{C}$ Mowat. ${ }^{1}$ Student, University of Dundee, Ninewells Hospital, Dundee, UK; ${ }^{2}$ Department of Colorectal Surgery, Ninewells Hospital, Dundee, UK; ${ }^{3}$ Department of Gastroenterology, Ninewells Hospital, Dundee, UK

10.1136/gutjnl-2014-307263.272
Introduction Symptoms associated with organic bowel disease such as cancer or adenomatous polyps are extremely non-specific. $^{1}$ Therefore, for individuals at moderate and high risk of colorectal cancer (CRC), the current practice involves surveillance colonoscopy. ${ }^{1}$ The BSG Guidelines for colorectal cancer screening and surveillance are a benchmark for UK clinicians. ${ }^{1}$ Approximately 2500 patients are under regular endoscopic surveillance in NHS Tayside; appointments are booked following a review and telephone consult by nurse specialists.

Methods Aim

To determine the level of adherence to the BSG guidelines, and the pathology findings from recent colonoscopies.

Methods Patients on the surveillance register who were reviewed between September 2012 and June 2013 were studied. Electronic data was retrieved from Unisoft, ICE and Clinical Portal to view colonoscopy reports, pathology findings and follow-up plans.

Results 434 patients were reviewed. 319 requests adhered to the guidelines (adherence 73.5\%), 328 patients (75.6\%) were scoped: 27 declined, 1 moved out with Tayside, 1 referred to genetics, 31 weren't required, 10 were unfit, 33 weren't due for colonoscopy whilst 3 patients postponed. 44 patient's colonoscopies fell out with the guidelines; 2 weren't due, 4 weren't required, with the rest (38) being brought back too early/late.

Median age 66 (range 21 - 96); Males 60\%. Females 40\%. Indication for surveillance was previous polyps (71\%), carcinoma $(11 \%)$, IBD $(4 \%)$ or a genetic family history $(14 \%)$. Colonoscopy identified normal bowel (58\%), polyps (40\%; 67\% of these were adenomas), IBD (2\%) and cancer (1\%).

Of 132 patients under 3 year follow-up for previous polyps; $54 \%$ had normal colonoscopy, 32\% had adenomas. Of 60 patients under 5 year follow-up for previous polyps, 67\% had normal colonoscopy, $18 \%$ had adenomas. Of 21 patients under 5 year follow-up for previous carcinoma $67 \%$ were normal, $19 \%$ had adenomas and there was one cancer. An additional 8 patients had a history of cancer within 3 years; 3 were found to have adenomas. Of 45 genetic family history patients $69 \%$ had normal colonoscopy, 18\% adenomas. Out of 13 IBD patients 7 had normal colonoscopy, 2 active IBD and 4 polyps with 1 being an adenoma.

Conclusion In Tayside the adherence to BSG guidelines was $73.5 \%$. The Nurse Specialist review saved a significant number of appointments. The majority of surveillance colonoscopies were normal; with the highest rate observed in those with a genetic family history. These findings suggest that alternative means of regular surveillance should be evaluated.

\section{REFERENCE}

1 Value of symptoms and additional diagnostic tests for colorectal cancer in primary care; systematic review and meta-analysis. BMJ 2010; 340:c1269doi:10.1136/ bmj.c1269

Disclosure of Interest None Declared.

\section{PWE-013 BOWEL CANCER SCREENING IN THE CZECH REPUBLIC : CURRENT STATUS, PROBLEMS, CLINICAL VIEW OF THE SINGLE MUNICIPAL SCREENING CENTRE AND THE NEWS IN 2014}

M Liberda*, A Bartkova. Gastroenterology, Valasske Mezirici Hospital, Valasske Mezirici, Czech Republic

\subsection{6/gutjnl-2014-307263.273}

Introduction Incidence of CRC in the Czech Republic (CR) $=7800-8200 /$ in population of $10 \mathrm{M}$ people. Approximately 